\title{
VIOLENCIA SIMBÓLICA CONTRA LA MUJER EN LOS ANUNCIOS PUBLICITARIOS: UN ANÁLISIS VISUAL CRÍTICO
}

\author{
Symbolic Violence Against Women in Advertising: A Critical Visual Analysis
}

VIOLENCE SYMBOLIQUE À L'ÉGARD DES FEMMES DANS DES ANNONCES PUBLICITAIRES: UNE ANALYSE VISUELLE CRITIQUE

\author{
María Martínez-Lirola \\ Doctora en Filología Inglesa, \\ Universidad de Sudáfrica y \\ Universidad de Alicante, España. \\ Profesora titular de Filología Inglesa, \\ Universidad de Alicante, España. \\ maria.lirola@ua.es \\ https://orcid. \\ org/0000-0002-6427-425X
}

\begin{abstract}
RESUMEN
El discurso publicitario es conocido por contribuir al sexismo y a la reproducción de estereotipos de género. Este artículo se centra en la violencia simbólica que presenta el cuerpo de las mujeres como objeto sexual en textos publicitarios empleados por Dolce \& Gabbana en la hoja informativa (newsletter) que envió, a través de la web, regularmente entre 2016 y 2019. La metodología utilizada fue fundamentalmente cualitativa, aunque se tuvieron en cuenta algunos datos cuantitativos para comprender mejor la estructura del corpus. Para deconstruir los significados representativos, interpersonales y composicionales de los textos se empleó la gramática visual de Kress y Van Leeuwen (2006). Los resultados del análisis visual muestran que los cuerpos de las mujeres son utilizados como reclamo publicitario, lo cual contribuye a la reproducción de varias formas de violencia simbólica. Tales resultados sugieren la necesidad de revisar la cosificación de los cuerpos femeninos en el discurso publicitario.
\end{abstract}

Palabras clave: mujeres; discurso publicitario; violencia simbólica; análisis visual crítico; estudios de género.

\section{Abstract}

Advertising discourse is known to contribute to sexism and to the reproductio of gender stereotypes. This research study focuses on the symbolic violence that presents the body of women as sexual objects in advertising texts used by Dolce $\&$ Gabbana in the newsletter that it sent through the web regularly between 2016 and 2019. The methodology is mainly qualitative, although some quantitative data is also provided to better understand the structure of the corpus. To deconstruct the representational, interpersonal and compositional meanings of the advertisements, the study uses Kress and van Leeuwen's (2006) visual grammar as a theoretical framework. The results of the visual analysis show that women's bod-

Recibido: 2020-05-05 / Aceptado: 2020-08-19 / Publicado: 2021-05-05

https://doi.org/10.17533/udea.ikala.v26n2a06 
ies are used as advertising "demands," which contributes to reproducing various forms of symbolic violence. Such results suggest the need to reconsider female body objectification in advertising discourse.

Keywords: women; advertising discourse; symbolic violence; critical visual analysis; gender studies.

\section{RÉSUMÉ}

Le discours publicitaire est connu par contribuer au sexisme et reproduire des stéréotypes de genre. Cet article se concentrera sur la déconstruction de la violence symbolique qui présente le corps de la femme comme un objet sexuel. Pour ce faire, les textes publicitaires utilisés par Dolce \& Gabbana dans leur fiche d'information (newsletter) qu'elle envoie régulièrement sur le web entre 2016 et 2019 ont été analysés. La méthodologie est fondamentalement qualitative, bien que certaines données quantitatives soient proposées pour mieux comprendre la structure du corpus. La grammaire visuelle de Kress et van Leeuwen (2006) sera utilisée comme cadre théorique pour déconstruire les significations représentatives, interpersonnelles et compositionnelles des textes. Les résultats montrent que le corps des femmes est utilisé comme une allégation publicitaire qui contribue à reproduire la violence symbolique. Une classification des principaux exemples de violence symbolique retrouvée dans le corpus sera proposée afin de bien faire comprendre la violence sexiste qui est encore employée. Les résultats indiquent la nécessité de réconsidérer la réifications des corps féminins.

Mots-clés : femmes; discours publicitaire ; violence symbolique ; analyse visuelle critique ; études de genre.

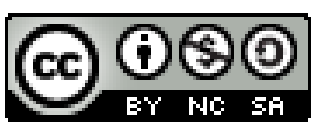




\section{Introducción}

El género está presente en todos los aspectos de la vida, incluida la publicidad, y el modelo que predomina es el que destaca la dominación masculina (Bourdieu, 1998, 2000). La influencia social de la publicidad es amplia, al tener un papel importante en la socialización de las personas, debido a que transmite modelos de comportamiento y pensamiento. En muchas ocasiones, un mensaje publicitario se acepta como el reflejo de los valores de una sociedad; como señala Bringas López (2010): "Los medios de comunicación, incluida la publicidad, son poderosos agentes de socialización que transmiten los códigos de conducta y modelos sociales de los grupos dominantes” (p. 117).

En la sociedad actual, el discurso publicitario es uno de los que más contribuye a la construcción de estereotipos de todo tipo, incluidos los de género, y a la transmisión de significados que, con frecuencia, se aceptan como reales (Ananda et al., 2019; Aparicio Martín, 2015; Buonanno, 2014; Sarkar, 2014). Por esta razón, es fundamental analizar las características de los textos publicitarios, por representar realidades sociales determinadas y por contribuir a la producción y la reproducción de la vida social, hecho que ocurre con todos los textos (Richardson, 2007).

Uno de nuestros principales intereses es estudiar textos en relación con el contexto en que se enmarcan, con el fin de establecer relaciones simbólicas entre los textos y la realidad social que les rodea. Es decir, nos proponemos analizar textos de la publicidad en los que se observe la representación que se hace de las mujeres, para entender cómo se construyen y qué ejemplos de violencia simbólica se asocian a la representación.

Las preguntas de investigación que se plantean en este estudio son las siguientes: ¿cómo se presenta a las mujeres en la publicidad de Dolce \& Gabbana? ¿Cuáles son las principales características visuales de los textos? ¿Hay ejemplos de violencia simbólica en los textos analizados? El análisis que se lleva a cabo para responder a estas preguntas nos permite poner de manifiesto que las diferentes elecciones visuales no son inocentes, sino que vienen determinadas por la ideología dominante y el contexto político-social que enmarca a los textos. Además, responder a estas preguntas nos permite entender cómo se proyecta a las mujeres como portadoras de elementos estéticos y conductas que configuran una imagen erótica de ellas reconocida colectivamente. También se observa el modo en que el cuerpo de las mujeres es empleado para seducir; de este modo, se fomenta un culto a la exhibición del cuerpo, que lleva a estereotipar los roles de género, uniendo a las mujeres al erotismo y a la seducción.

La hipótesis de partida es que la publicidad, en general, y la de Dolce \& Gabbana, en particular, utiliza más los cuerpos de las mujeres que los de los hombres para publicitar sus productos y recurre a la violencia simbólica en la representación. Por esta razón, los objetivos de este artículo son: 1) observar ejemplos de violencia simbólica en una muestra de discurso publicitario, en concreto, en los textos que Dolce \& Gabbana utiliza en su hoja informativa (newsletter); y 2) establecer una clasificación de los principales tipos de violencia simbólica encontradas en los textos.

Dolce \& Gabbana es una firma de moda italiana fundada por Domenico Dolce y Stefano Gabbana en el año 1985. Esta firma ha sido seleccionada por su popularidad a nivel mundial y por la gran variedad de artículos que vende: ropa, gafas, complementos, perfumes, móviles, restaurantes, entre otros. Es decir, se ha optado por seleccionar una firma que publicita varios tipos de productos, con el fin de observar ejemplos de violencia simbólica, con independencia del producto que se publicite.

Este artículo se divide en las siguientes secciones: a continuación, se ofrece un marco teórico; seguidamente, se enuncian los datos y la metodología; luego, se presenta el análisis del corpus, donde se muestran algunos datos cuantitativos y se ilustran 
los principales tipos de violencia simbólica encontrados con el análisis cualitativo de tres textos. El artículo finaliza con la discusión y las conclusiones del estudio, donde se señala que la publicidad sexista sigue estando presente en el siglo XXI.

\section{Marco teórico}

El mensaje publicitario es una práctica discursiva que se emplea con el fin de persuadir, informar, captar la atención, etc. Se trata de un tipo de discurso que utiliza distintas técnicas para manipular y persuadir a la compra de productos, a adoptar un estilo de vida, etc. (Cruz García, 2017; Danilina et al., 2019). En este sentido, las mujeres son normalmente las utilizadas como estímulos en la publicidad (Sato, 2011) y su representación viene determinada por normas culturales (Jeffries, 2007).

El discurso de la publicidad visibiliza a las mujeres y utiliza constantemente sus cuerpos como reclamo publicitario, lo que hace que la mujer se defina, en general, en función de su cuerpo, sin tener en cuenta sus características intelectuales, lo que, por el contrario, llevaría a considerar su integridad como ser humano, sin conceder importancia exclusiva a los aspectos físicos, entendidos como marca de su valor social.

Son diversos los estudios que han prestado atención a cuestiones relacionadas con la lengua empleada en la publicidad y el género (Cameron, 2006; Coates, 2004, 2012; Eckert y McConnellGinet, 2003; Goddard y Patterson, 2000; Hashim y Hassan, 2011; Talbot, 2010; Wodak, 1997, entre otros). Además, son múltiples los estudios que han prestado atención a la lengua visual y a cuestiones de género relacionadas con las representaciones de los cuerpos de las mujeres (Baykal, 2016; Bowcher, 2012; Fernández Fernández et al., 2014; Jeffries, 2007; Jerslev, 2017; Magalhães y Caetano, 2012; Martínez Lirola, 2012a y 2012b; Martínez Lirola y Chovanec, 2012; Sato, 2011; Sharda, 2014; Velandia-Morales y Rincón, 2014; Xu y Tan, 2020, entre otros). Los estudios coinciden en que las mujeres y los hombres son representados de forma distinta en la publicidad, debido al predominio del cuerpo de las mujeres como reclamo publicitario (Del Rosso, 2017; Giaccardi et al., 2016; Mora, 2014; Shaikh et al., 2016). En este sentido, concurrimos con Verdú Delgado (2018) en que

La difusión masiva de una imagen de la feminidad basada de nuevo en la asociación de mujeres y sexualidad, invisibilizando el resto de dimensiones e intereses que dan sentido a la vida humana, mantiene sin duda un imaginario social útil para justificar la no consideración de las mujeres como sujetos de derecho en una época en la que no es posible emplear aparatos institucionales para dicha causa sin cuestionamientos (p. 168).

Al estudiar el modo en que las mujeres aparecen representadas en los textos publicitarios, se observa que el atractivo sexual (belleza, hermosura, sex appeal o deseabilidad) es empleado de manera recurrente para reclamar la atención y persuadir (Galarza et al., 2016; Gil Calvo, 2000). Así, los cuerpos de las mujeres se usan como reclamo publicitario, por medio de posturas, escotes o cosmética, sin tomar realmente conciencia de las repercusiones sociales que estas representaciones pueden tener. En pocos estudios se analizan las formas de violencia simbólica asociadas a concebir el cuerpo como objeto de deseo y portador de placer. Además, el cuerpo femenino es el lugar donde tienen lugar diversas formas de poder (Muñiz, 2010; Pham, 2014). La imagen y el cuerpo de las mujeres reflejan los valores de la sociedad; el ideario colectivo entiende que el objetivo del cuerpo femenino es agradar y adaptarse a una norma imaginaria (Walter, 2010).

Las ideas enunciadas en el párrafo anterior se relacionan con la violencia simbólica. Esta tiene lugar sin coacción física, por medio de formas simbólicas que implican que tanto dominantes como dominados se asumen en ese papel sin apenas cuestionarlo (Bringas López, 2010; Martín Lucas, 2010). La violencia simbólica está presente en mensajes o signos que reproducen relaciones de dominación y marginación contra las mujeres en la sociedad en general y en la publicidad en particular. A veces se entiende, de manera inconsciente, 
como parte de la normalidad y de la cotidianidad, por lo que no se la considera dañina o amenazante. Los ejemplos de violencia simbólica señalan que los estereotipos patriarcales creados sobre las mujeres siguen estando presentes en la actualidad.

Además, la violencia simbólica va unida a mensajes, ideas o valores que reproducen o manifiestan marginación, subordinación o dominación entre personas. Blanco (2009) considera que toda forma de violencia de género es violencia simbólica, porque en ella se observan las desigualdades entre mujeres y hombres, que han estado presentes a lo largo de la historia; estas afectan a los cuerpos, por los significados culturales y sociales que se asocian con ellos. En palabras de Blanco (2009): "Esta construcción social del cuerpo por la cual atraviesa todo ejercicio de dominación simbólica, se da en una interrelación entre aspectos como la etnia, el sexo, la lengua y la religión” (p. 64).

Generalmente, la violencia simbólica se caracteriza por ser sutil y por ser una forma de ejercer poder, como señala Bourdieu (2000): "La violencia simbólica es una forma de ejercer el poder, que repercute directamente sobre el cuerpo de la persona, como por arte de magia, sin restricciones físicas aparentes" (p. 44). El mismo autor profundiza en cómo esta forma de violencia afecta a los cuerpos:

La fuerza simbólica es una forma de poder, que se ejerce directamente sobre los cuerpos y como por arte de magia, al margen de cualquier coacción física; pero esta magia sólo opera apoyándose en unas disposiciones registradas, a la manera de unos resortes, en lo más profundo de los cuerpos (Bourdieu, 2000, p. 54).

Las estructuras simbólico-culturales invisibilizan, en ocasiones, la violencia simbólica que se ejerce contra las mujeres. En este sentido, muchas manifestaciones de violencia simbólica se perciben como normales y son aceptadas de manera legítima. Además, el poder simbólico determina las representaciones simbólico-culturales que se hace de las mujeres en la sociedad actual.

Hemos elegido la perspectiva que propone el análisis crítico del discurso (ACD) para llevar a cabo el estudio de los textos seleccionados, por tener aquel como uno de sus fines fundamentales establecer relaciones entre el discurso y la sociedad; de ahí que se centre en analizar textos reales, con el fin de deconstruir la ideología y el poder presentes en los textos. El ACD no solo describe la sociedad a través de los textos, sino que también trata de modificarla (Van Dijk, 2009; Wodak, 2011). El ACD visibiliza las relaciones de poder y desigualdad que se observan en la sociedad a través de las características de los textos (Van Leeuwen, 2008).

Mi interés en el ACD como perspectiva viene determinado por la importancia que este presta a la deconstrucción ${ }^{1}$ de estructuras de discriminación, dominación, poder o control que se presentan en algunas situaciones sociales o con determinados grupos, en este caso con las mujeres, en el discurso publicitario. Analizar las características visuales de los textos que forman el corpus nos permite entender el modo en que se representa a las mujeres en el discurso publicitario empleado por Dolce \& Gabbbana y en qué medida ese discurso construye la realidad social. En palabras de Wodak (2011): "Discourse is socially constitutive as well as socially shaped: it constitutes situations, objects of knowledge and the social identities of and relationships between people and groups of people" (p. 39).

El ACD permite profundizar en cuestiones de género, entre las que destacan el sistema de dominación que los hombres ejercen, en muchas ocasiones, sobre los cuerpos de las mujeres, o el valor social asociado a las mujeres por medio de la belleza de sus cuerpos, hecho en el que ella participan influidas por el dominio patriarcal que en ellas se ha impuesto (Perdomo Colina, 2019; SoleyBeltran, 2015). En este sentido, el ACD contribuye a desenmascarar los códigos de belleza asociados a la construcción de la feminidad. Por tanto, la perspectiva del ACD es de utilidad para deconstruir

1 Esta se define como el desmontaje de un concepto o de una construcción intelectual por medio de su análisis, mostrando así contradicciones y ambigüedades (Diccionario online de la Real Academia Española). 
cuestiones de género, entre las que se encuentran algunos planteamientos importantes para este estudio: el cuerpo de las mujeres es una construcción social, el ideal de belleza contribuye a perpetuar estereotipos de género que afectan más a las mujeres que a los hombres, y la pervivencia de modelos de publicidad sexista, en los que predomina el empleo del cuerpo de las mujeres como reclamo publicitario.

Finalmente, el hecho de trabajar con textos en los que la imagen es el elemento principal hace necesario que se emplee el modelo de gramática visual propuesto por Kress y Van Leeuwen (2006). Su método de semiótica social se centra en analizar cómo la lengua y otros modos de comunicación funcionan para construir significados; además, este modelo asigna significados representativos (cómo las realidades se representan en los textos), interpersonales (las relaciones sociales entre los personajes y con los lectores) y compositivos (la forma en que los textos son coherentes y establecen relaciones con su contexto) a imágenes, basados en la semiótica social de Halliday (1978) y el modelo de tres metafunciones (ideacional, interpersonal y textual) propuesto por Halliday y Matthiessen (2004).

La metafunción de representación presta atención a: participantes (quién y qué se representa), procesos (acciones o relaciones representadas) y circunstancias (dónde, cuándo, cómo, con qué). Los vectores contribuyen a crear dinamismo o acción entre los diferentes participantes. La apariencia física de los personajes ayuda a los lectores a inferir su edad, etnia, clase y papel.

Teniendo en cuenta los significados interpersonales, Kress y Van Leeuwen (2006) distinguen tres tipos de sistemas asociados a la función interpersonal: los de 1) acto y mirada de imagen, 2) distancia social e intimidad, y 3) participación y poder.

En cuanto a la imagen y la mirada, la imagen de los personajes es "petición" (demand) cuando los personajes miran al espectador. Por el contrario, la imagen es "ofrecimiento" (offer) cuando los personajes no miran a la audiencia.
La distancia social se concentra en la representación de los personajes como cerca, a media distancia y a larga distancia.

La participación y el poder se relacionan con observar a las personas representadas desde distintos ángulos y analizar cómo estos contribuyen a posicionar al espectador: el ángulo horizontal implica una mayor o menor implicación, es decir, cuando los personajes se presentan frente a los espectadores, están involucrados con la audiencia, mientras que su representación como un ángulo oblicuo contribuye a separarlos de los lectores. El ángulo vertical está asociado con la realización del poder: si los espectadores miran hacia arriba, se les da poder, mientras que lo que se mira hacia abajo, parece débil y vulnerable (Kress y Van Leeuwen, 2006, p. 140 ss).

La función composicional se concentra en el valor de la información, el elemento más sobresaliente y los marcos (Kress y Van Leeuwen, 2006). El valor de la información permite una profundización de la ubicación y la relación entre los elementos: colocar elementos a la derecha (nueva información) y a la izquierda (información conocida) contribuye a dar más importancia a ciertos elementos. Además, los elementos de la parte superior de la presentación funcionan como "ideales", mientras que los de la posición inferior funcionan como "reales". En cuanto a la prominencia, se centra en los elementos que se destacan por su tamaño, color, enfoque, etc. Los marcos muestran si los elementos van o no van unidos.

\section{Método}

El análisis se centró en los textos publicitarios empleados por Dolce \& Gabbana en su hoja informativa (newsletter) que envía regularmente mediante la web. El corpus consiste en un total de 272 textos. Se analizaron todos los textos presentes en dicha hoja informativa desde el 1 de enero del año 2016 hasta el 31 de diciembre del año 2019.

Una vez recopilados los textos, se estudiaron sus principales características visuales, desde un punto 
Tabla 1 Los actores sociales en los textos analizados.

\begin{tabular}{lccccc}
\hline Años & Número de textos & $\begin{array}{c}\text { Solo una mujer } \\
\text { representada (\%) }\end{array}$ & $\begin{array}{c}\text { Solo un hombre } \\
\text { representado (\%) }\end{array}$ & $\begin{array}{c}\text { Sin representación } \\
\text { humana (\%) }\end{array}$ & $\begin{array}{c}\text { Más de una persona } \\
\text { representada (\%) }\end{array}$ \\
\hline 2016 & 34 & 36,36 & 0 & 18,18 & 45,46 \\
2017 & 79 & 44,41 & 2,53 & 32,91 & 20,24 \\
2018 & 120 & 32,52 & 4,16 & 41,66 & 21,66 \\
2019 & 39 & 38,46 & 0 & 35,89 & 25,64 \\
Total & 272 & & & & \\
\hline
\end{tabular}

de vista crítico discursivo, prestando atención: 1) a la función representacional, al examinar quiénes son los actores sociales ${ }^{2}$ representados; 2) a la interpersonal, al ocuparse de las relaciones entre las personas representadas y la audiencia; y 3) a la composicional, al analizar el valor de la información, el rasgo más sobresaliente y los marcos. Seguidamente, se revisaron ejemplos de violencia simbólica asociados con mujeres en los textos analizados y se estableció una clasificación que sistematiza los principales tipos de violencia simbólica encontrados (véase el siguiente apartado).

La metodología es fundamentalmente cualitativa-descriptiva, pero en la descripción general del corpus también se utilizan algunos datos cuantitativos, con el fin de ofrecer una descripción lo más completa posible de los textos analizados.

Debido a las limitaciones de espacio de este artículo, se ofrece un análisis detallado de tres textos que ilustran las principales características de los tipos de violencia simbólica encontrados en el corpus.

\section{Análisis cuantitativo del corpus}

Este apartado se divide en dos subsecciones: inicialmente, se presentan las principales características cuantitativas del corpus de textos analizados. Luego, debido a las limitaciones de espacio de este artículo, se ofrece un análisis cualitativo, siguiendo la tradición del ACD, de tres textos que son ejemplos claros de los principales tipos de violencia simbólica observados en el corpus.

2 Personas que aparecen en los textos.

\section{Análisis cuantitativo del corpus}

Antes de ocuparnos de los textos que ilustran los principales tipos de violencia simbólica encontrados en el corpus, vamos a señalar algunas cuestiones generales del corpus, relacionadas con la función representacional (véanse Tablas 1 y 2), con la función interpersonal (véase Tabla 3) y con la función composicional (véase Tabla 4).

Tabla 2 Representación racial en los actores sociales

\begin{tabular}{lcccc}
\hline Años & $\begin{array}{c}\text { Número } \\
\text { de textos }\end{array}$ & $\begin{array}{c}\text { Raza } \\
\text { blanca (\%) }\end{array}$ & $\begin{array}{c}\text { Mezcla } \\
\text { racial (\%) }\end{array}$ & $\begin{array}{c}\text { Personas } \\
\text { de raza } \\
\text { negra (\%) }\end{array}$ \\
\hline 2016 & 34 & 90,91 & 9,09 & 0 \\
2017 & 79 & 94,94 & 5,06 & 0 \\
2018 & 120 & 90,84 & 7,5 & 1,66 \\
2019 & 39 & 87,19 & 7,69 & 5,12 \\
Número & 272 & & & \\
total & & & & \\
\hline
\end{tabular}

Tabla 3 Representación de los cuerpos y significados interpersonales

\begin{tabular}{lcccc}
\hline Años & $\begin{array}{c}\text { Número } \\
\text { de textos }\end{array}$ & $\begin{array}{c}\text { Cuerpo } \\
\text { cortado } \\
(\%)\end{array}$ & $\begin{array}{c}\text { Petición } \\
\text { (demand) } \\
(\%)\end{array}$ & $\begin{array}{c}\text { Ofrecimiento } \\
\text { (offer) } \\
(\%)\end{array}$ \\
\hline 2016 & 34 & 54,54 & 63,63 & 36,37 \\
2017 & 79 & 49,36 & 34,17 & 65,83 \\
2018 & 120 & 42,5 & 62,50 & 37,50 \\
2019 & 39 & 46,15 & 46,15 & 53,85 \\
Número & 272 & & & \\
total & & & & \\
\hline
\end{tabular}


Tabla 4 Posición del producto en la publicidad de Dolce \& Gabbana

\begin{tabular}{lcccc}
\hline Años & $\begin{array}{c}\text { Número } \\
\text { de } \\
\text { textos }\end{array}$ & $\begin{array}{c}\text { Producto a } \\
\text { la derecha } \\
(\%)\end{array}$ & $\begin{array}{c}\text { Producto } \\
\text { en el } \\
\text { centro (\%) }\end{array}$ & $\begin{array}{c}\text { Producto a } \\
\text { la izquierda } \\
(\%)\end{array}$ \\
\hline 2016 & 34 & 90,91 & 9,09 & 0 \\
2017 & 79 & 21,51 & 59,51 & 18,98 \\
2018 & 120 & 13,33 & 84,17 & 2,5 \\
2019 & 39 & 2,56 & 94,88 & 2,56 \\
Número & 272 & & & \\
total & & & & \\
\hline
\end{tabular}

En la función representacional es fundamental observar quiénes son los participantes que intervienen en los textos. De los 272 textos producidos en los cuatro años, en los textos predomina la presencia de mujeres frente a hombres, en todos los casos. Es destacable que, en 2017, las mujeres aparecen en casi la mitad de los textos analizados, en concreto en el 44,41\%, frente a un 2,53\% de textos en los que se representa solo un hombre. En los otros tres años, también destaca la presencia femenina y hemos de señalar que ni en 2016 ni en 2019 aparecen representados hombres solos.

Cuando se opta por representar a más de una persona en los textos, normalmente se publicita más de un producto. Destaca que, en 2016, casi un $50 \%$ de los textos, en concreto el 45,46\%, representen a más de una persona. El porcentaje en los otros tres años es menor.

Hemos de señalar que también hay textos en los que se no hay representación humana, de modo que el producto ocupa el lugar central. El mayor porcentaje de este tipo de textos se da en 2018, el 41,66\%, año en que se publicaron menos textos solo con mujeres, seguido del 35,89 \% del año 2019. En 2016, el porcentaje de textos en los que aparece solo el producto es escaso, $18,18 \%$, por ser este el año en que hay más textos con varias personas.

Las mujeres representadas destacan por su belleza. Se trata de mujeres jóvenes, delgadas, bien vestidas y la gran mayoría son de raza blanca, como se muestra en la Tabla 2. Aunque hay textos en los que se exhibe mezcla racial en los distintos años analizados, en ningún caso se llega al $10 \%$. Al observar los textos en los que aparecen solo personas de raza negra, es muy significativo que hay dos años en los que no hay ejemplos, y cuando los hay, solo son un porcentaje muy reducido, cuyo valor máximo es 5,12\%, en 2019.

$\mathrm{Al}$ prestar atención a la función interpersonal destaca que los cuerpos aparezcan cortados es un elemento que contribuye a determinar la violencia simbólica, debido a que no se presenta a las personas en su totalidad, como seres íntegros, hecho que dificulta establecer una relación entre los actores sociales representados y la audiencia. Los ejemplos en los que los actores sociales interaccionan, al ser ejemplos de petición, por mirar de forma directa y, por lo tanto, pedir una respuesta, predominan tanto en 2016 (63,63\%) como en 2018 (62,5 \%). Esto contrasta con los otros dos años, donde hay más ejemplos de representación de las personas como ofrecimiento: $65,83 \%$ en 2017 y $53,85 \%$ en 2019 , pues no hay contacto visual con la audiencia, hecho que sugiere la idea de que son personas objeto para ser observadas.

En la función composicional es importante prestar atención al lugar del texto en que se ubica el producto que se quiere vender. $\mathrm{Al}$ analizar la posición del producto (véase Tabla 4), llama la atención que, en 2016, la tendencia sea a situarlo a la derecha, el lugar de la posición más importante en los textos en el 90,91 \% de los casos. En los otros tres años, la tendencia es a situar los productos en el centro, especialmente en 2019, con el 94,88\% de los textos en esa posición, al ser esta una que contribuye a destacar el producto. Otro dato destacable en esta parte del análisis es el hecho de que, en 2017, es similar el número de textos en los que el producto aparece a la derecha $(21,51 \%)$ y a la izquierda $(18,98 \%)$.

\section{Análisis cualitativo del corpus}

Este apartado se subdivide en tres secciones, que coindicen con los tres modos principales de violencia simbólica observados en los textos estudiados. 
Debido a las limitaciones de espacio de este artículo, cada uno de los tipos de violencia manifiestos en el corpus de ejemplos se ilustra con un texto que es representativo de ese tipo de violencia. El análisis de las tres metafunciones permite profundizar en la representación de los cuerpos y señalar algunos ejemplos de violencia simbólica contra las mujeres, al identificar la postura, la posición o el movimiento de los cuerpos. El erotismo y la sensualidad que se expresan en los textos también contribuyen a utilizar los cuerpos femeninos como reclamo publicitario, hecho que igualmente se relaciona con la violencia simbólica.

Se espera que futuras investigaciones presenten el análisis de otros textos que sirvan para seguir ilustrando los tipos de violencia que hemos sistematizado. Esperamos que la clasificación que ofrecemos sirva para estudiar otros textos publicitarios y para detectar los casos de violencia simbólica que se hallan en el discurso publicitario, que no contribuyen al empoderamiento de las mujeres.

\section{El cuerpo como reclamo publicitario dinámico, a través del movimiento, la desnudez y el erotismo}

El texto publicitario de Dolce \& Gabbana de 2016 (ver enlace) $)^{3}$ sirve irve para ilustrar uno de los tipos de violencia simbólica encontrados en el corpus: se trata del uso del cuerpo de las mujeres como reclamo publicitario, a través del movimiento, la desnudez y el erotismo.

En la función representacional destaca el hecho de que, en el texto, hay doce hombres frente a cuatro mujeres (uno es un cura). Los hombres destacan por ir vestidos con elegancia y por permanecer casi estáticos, con la excepción de dos que aparecen bailando con dos de las mujeres representadas. Esto contrasta con el dinamismo y el movimiento de

3 Con el ánimo de respetar los derechos de autor de la marca Dolce \& Gabbana, se evita reproducir la imagen y se dejan solo los enlaces consultados. Vea el enlace aquí: https://i.blogs.es/24a87e/campanas-de-moda-primavera-verano-2016-trendencias-hombre-2/650_1200.jpg las dos mujeres representadas en el centro, claramente activas, al mover sus cuerpos con erotismo y sensualidad, algo similar a lo que ocurre con la bailarina de la derecha con la diferencia de que lleva ropa menos provocativa.

La acción tiene lugar en el exterior de un restaurante y se observan distintos vectores de acción, que sirven para unir a los actores sociales representados, entre los que destacan los brazos, las piernas y las caderas de las dos mujeres de rojo. La pasividad de la mayoría de los hombres representados contrasta con el dinamismo y el movimiento de las dos mujeres en el centro, de las dos parejas que bailan y de la señora a la izquierda que aplaude mientras sonríe.

Al considerar la función interpersonal, se observa que tanto las dos mujeres que destacan como seis de los hombres miran a la audiencia, de modo que demandan una respuesta (demand) o entran en una relación simbólica con la audiencia (Kress y Van Leeuwen, 2006, p. 118). Esto contrasta con el resto de personas que se miran entre sí, es decir, representan un contacto visual entre unos y otros, sin establecer contacto con la audiencia, de modo que se trata de un ejemplo de ofrecimiento (offer).

La distancia social que se expresa en el texto es clara entre las/os lectoras/es y los actores sociales representados, al aparecer la mayoría en segundo y tercer plano. Esto contribuye a que la escena que se presenta, en la que destaca la pose erótica de las dos mujeres, sea observada por la audiencia desde la distancia. Esto contrasta con el ángulo horizontal, que sí contribuye a establecer relaciones entre los personajes y la audiencia. Con respecto al ángulo vertical, este contribuye a destacar a los personajes y a darles poder sobre la audiencia, especialmente en las dos mujeres de rojo que miran a las/os lectoras/es desde arriba.

Con respecto a la función composicional, en el valor de la información, nos encontramos ante un texto que se lee del centro a los márgenes, debido al hecho de que la mayoría de los actores sociales están en el centro y a que las dos mujeres están de 
pie encima de las mesas en la posición central. El hecho de que las dos mujeres estén localizadas en el centro, con poca ropa, vestidas de rojo, contribuye a presentar el cuerpo como reclamo publicitario y como ejemplo de violencia simbólica, al contrastar claramente el modo en que se representan estas dos mujeres con el resto de personas del texto.

Estas dos mujeres son el elemento más sobresaliente no solo por su posición, sino también por la ropa que llevan, en la que predomina el color rojo, que les permite mostrar las formas de su cuerpo y por su postura sensual. Además, ubicar estas dos mujeres en la parte superior del texto contribuye a destacarlas como "ideales", en contraste con las demás personas en la parte baja del texto, cuya posición aporta a presentarlas como "reales".

Los actores sociales están enmarcados por el toldo rojo que se halla en el fondo de la imagen, cuyo color sirve para destacar aún más la ropa de las mujeres. Además, las personas representadas en el centro están enmarcadas por otras dos mujeres, que aparecen una a la derecha y otra a la izquierda en primer plano, cuya ropa contrasta con las de las dos mujeres del centro, no solo por el color (blanco y negro), sino también porque cubre casi todo su cuerpo. El enmarcamiento no solo es por las mujeres, sino que si va más allá, es por los bailarines. El de la izquierda es un cura es destacable que no se ve la cara de la acompañante con quien baila, sino que se representa que es del mismo nivel social que la bailarina de blanco por la bufanda.

\section{El cuerpo como reclamo publicitario estático a través de la postura: cuerpos-escaparate}

El texto de la campaña de Dolce \& Gabbana de $2019^{4}$ sirve para ilustrar otro tipo de violencia simbólica encontrada en el corpus: se trata de emplear el cuerpo como escaparate, al estar estático para publicitar los productos de la firma. Las mujeres muestran una imagen perfecta, al ser representadas posando con unos trajes elegantes de la firma.

4 https://pbs.twimg.com/media/D8zAK0LWkAUFjPA. jpg
$\mathrm{Al}$ analizar la función representacional, lo primero destacable es que encontramos cuatro actores sociales, tres mujeres y un hombre. Ellas aparecen estáticas, están bien vestidas y maquilladas; muestran su cuerpo sin movimiento, lo que nos permite hablar del cuerpo como escaparate, que sirve para presentar a la audiencia los productos que se quieren vender. Las mujeres posan, miran al espectador y muestran su imagen física Esto contrasta con el hombre que ubica al final del texto, al que se representa haciendo una postura dinámica al ser un equilibrista.

Se observan distintos vectores de acción que unen a los cuatro actores sociales a través de la cabeza o la boca, entre otros. Los brazos en jarra de la mujer que aparece en tercer lugar sirven para establecer una relación entre ella, las otras dos mujeres y el hombre que está a la derecha y en tercer plano. La acción de posar y de hacer la postura acrobática tiene lugar en la calle. Además de por vectores, la primera y la tercera mujer están conectadas también al llevar una ropa de un color similar, que también está en el equilibrista.

En la función interpersonal, una de las características que más destaca es el hecho de que las tres mujeres miran directamente a la audiencia, con la que establecen una relación simbólica de demanda. La distancia social entre los distintos actores sociales y la audiencia varía, debido a que encontramos una mujer en primer plano, dos en segundo y el único hombre representado aparece en tercer plano. La mujer que está en primer plano se presenta muy próxima a la audiencia, hecho que contribuye a proyectar una relación cercana. Además, el ángulo horizontal contribuye a facilitar la relación entre esta mujer y las dos que se encuentran en segundo plano con las/os lectoras/es. El ángulo vertical ayuda a destacar a las tres mujeres representadas, que aparecen ante la audiencia empoderadas gracias a los vestidos elegantes que llevan puestos.

El valor de la información de este texto rompe el principio de composición por el que normalmente a la derecha aparece la información nueva 
y más importante (Kress y van Leeuwen, 2006). A la derecha y en tercer plano está el hombre haciendo una acrobacia. El equilibrista no tiene "reclamo" alguno con la audiencia, pero sí está en cierta forma señalando con los pies en otra dirección contraria a la de las mujeres, o incluso se forma un "ángulo" entre las mujeres y la posición de los pies del equilibrista. En la perspectiva que contextualiza la visual se observa que se va de un primer plano a uno más profundo, donde al final el equilibrista corta y redirige la visual. El nombre de la firma se ubica a la izquierda en blanco, hecho que contribuye a destacarla. Sin embargo, en este texto la información más importante está del centro a la izquierda, que es donde se hallan las mujeres. El elemento más relevante del texto es la mujer a la izquierda, pues está en primer plano y su tamaño es mayor. Todos los actores sociales están enmarcados por el edificio y el monumento que se observa. Destaca que los adornos que aparecen en la cabeza de las mujeres en cierto modo se relacionan con la hoja que sale del monumento, y con los pies del equilibrista, que también están sobre la cabeza. Finalmente es destacable el "corte de cuerpo" de las mujeres pues la imagen combina cuerpo cortado con cuerpo completo. Además, hay una secuencia en las vestimentas de las mujeres que va de cubierto a descubierto.

\section{El cuerpo cortado para destacar y enmarcar el producto que se publicita}

En la imagen de Dolce \& Gabbana de 2017,5 el texto ilustra un ejemplo de violencia simbólica muy común en la publicidad de Dolce \& Gabbana. Se trata de un caso claro en que no se representa el cuerpo de la mujer en su totalidad, sino que es solo una parte, en este caso la cabeza (en otros textos se emplea solo el busto para enmarcar el producto). Presentar el rostro de la mujer al mismo nivel que el producto contribuye a su instrumentalización y a emplear el cuerpo como reclamo publicitario.

5 https://i.mdel.net/i/db/2017/1/632906/632906800 w.jpg
El rostro de la mujer muestra que es joven y blanca; el color de sus ojos también es similar al color del bote del perfume que se publicita, y el vector entre los ojos y el tapón del bote sirve para establecer esta relación. Además, el hecho de que la imagen esté enmarcada en el mar también contribuye a establecer relaciones entre el perfume que se publicita y el contexto de la imagen. El hecho de que la mujer mire a la audiencia indica que es utilizada como reclamo publicitario.

Nos encontramos ante un ejemplo claro de petición (demand), pues la mujer mira directamente a la audiencia, hecho que puede interpretarse como una petición para que compre el perfume situado a la derecha. La distancia social entre el producto, la mujer representada y la audiencia es corta; además, el ángulo horizontal contribuye a implicar a la audiencia con el texto, al tratarse de un ángulo frontal. El ángulo vertical también permite a las/ os lectores/as acercarse al producto.

El valor de la información muestra que nos encontramos ante un ejemplo en el que el producto aparece a la derecha, el lugar de la información nueva que se quiere destacar, mientras que la mujer es ubicada a la izquierda, el lugar de la información conocida y menos importante, hecho que contribuye a que no tenga protagonismo en el texto, sino que su presencia se justifica por ser utilizada como reclamo publicitario. Sin embargo, ella es el elemento más sobresaliente en el texto, por su tamaño. El enmarque es distinto en la mujer y en el producto. La mujer aparece con el fondo marino, cielo y roca, mientras que el producto solo sobresale sobre la roca. Se observa una "montaña hueca", por la que se ve el mar y el cielo.

\section{Discusión}

El análisis de los textos llevado a cabo nos introduce en un mundo semántico y simbólico complejo, el de los textos publicitarios, cuyo fin es mostrar a las mujeres siguiendo las expectativas de la ideología patriarcal dominante, hecho que lleva consigo la normalización de distintas formas de violencia 
simbólica contra las mujeres. Así, los ejemplos de violencia simbólica estudiados en el corpus contribuyen a desenmascarar el abuso, el poder y la dominación hacia las mujeres, pues el cuerpo se utiliza como reclamo publicitario y como objeto sexuado; de ahí la preferencia por mujeres jóvenes, delgadas y guapas. En este sentido, se observan relaciones entre el análisis cuantitativo y el cualitativo presentados en este artículo, pues en ambos se enuncia que los cuerpos de las mujeres son hipervisibles, tanto por el número de veces que aparecen, como por la posición en los textos, aspectos que sin duda contribuyen a emplear el cuerpo femenino como reclamo publicitario.

Además, ambos análisis muestran que la presencia masculina es escasa, aunque hay hombres en los textos en que aparecen varias personas representadas. En consecuencia, se exhiben a la audiencia textos sexistas, en los que se observa una clara asimetría entre la representación de mujeres y de hombres. Esto se enfatiza aún más por el hecho de que las mujeres utilicen ropa provocativa que contribuye a marcar sus formas corporales. En definitiva, tanto el análisis cuantitativo como el cualitativo muestran que las mujeres y los hombres no se representan de la misma manera en los textos estudiados, hecho que se relaciona con la desigualdad que afecta a las mujeres en muchos aspectos, incluida la publicidad.

Las características de la gramática visual empleadas para mostrar el cuerpo como representación social en el corpus permiten deconstruir los textos y desenmascarar violencias simbólicas que contribuyen a cosificar el cuerpo, entre las que destacan: la erotización del cuerpo de la mujer, al presentarlo como deseable; el empleo del cuerpo como escaparate, o la representación parcial del mismo, al aparecer el cuerpo cortado.

Exponer a las mujeres como objeto sexual es un ejemplo claro de violencia simbólica (texto 1), al contribuir a la instrumentalización del cuerpo, cuyo fin principal es emplearlo para vender productos de la firma. Presentar los cuerpos como deseables y erotizados, al destacar las zonas erógenas o al seleccionar posturas sexis y sensuales, contribuye a sexualizarlo. De esta forma, los cuerpos se convierten en objeto, siguiendo las normas del sistema patriarcal que nos enmarca, y que dicta pautas de acción y comportamiento de manera directa e indirecta a la sociedad. La ideología patriarcal contribuye a normalizar que los cuerpos de las mujeres se adapten a los cánones establecidos como ideal de belleza, con el fin de agradar a los hombres o de servir de reclamo publicitario.

El hecho de que los cuerpos de las mujeres representadas aparezcan cortados es un ejemplo claro de violencia simbólica (textos 2, 3), pues la fragmentación de los cuerpos lleva consigo que hay determinadas zonas, normalmente zonas erógenas, que se emplean para enmarcar los productos que se publicitan. Este tipo de violencia simbólica se consolida como una forma de dominación sobre los cuerpos de las mujeres. En consecuencia, el discurso de la publicidad, en este caso el de Dolce \& Gabbana, contribuye a reproducir el poder social del sistema patriarcal, al utilizar los cuerpos para destacar, enmarcar o decorar los productos que se quieren vender.

El empleo del cuerpo como escaparate (textos 1, 2 y 3) contribuye al uso de las mujeres como reclamo publicitario, sin duda otro ejemplo de violencia simbólica. En los tres casos, el cuerpo es utilizado para captar la atención, adorado como portador de belleza y erotismo, que sirven para provocar la atracción sexual y el deseo, sin hacer ninguna referencia a las capacidades intelectuales de las mujeres. Así, se construye una ideología sobre el cuerpo, en la que lo que prima es la belleza, la juventud y la evocación de la sexualidad.

La cosificación y las violencias invisibles observadas en los textos no favorecen el respeto al cuerpo o a la igualdad en el tratamiento de la representación de los cuerpos de las mujeres y los hombres en la publicidad. Por esta razón, es fundamental reivindicar la importancia de la mujer en su totalidad, sin centrarse solo en el cuerpo como elemento fundamental en su identidad (Martínez Lirola, 2009, 2010, 2012a). 
En general, no se profundiza en las realidades sociales de las mujeres representadas, sino que lo que se fomenta es una lectura superficial, que se centra en la belleza de sus cuerpos como portadores de sexualidad. Los cuerpos se conciben como pasivos, receptores de las miradas de otros, como objetos deseables, que pueden utilizarse para seducir, vender o persuadir. En este sentido, el cuerpo se instrumentaliza con objetivos marcados por el grupo en posición de poder, por el sistema patriarcal que nos enmarca. Esta representación vulnera la "Disposición Adicional Sexta" de la Ley orgánica de medidas de protección integral contra la violencia de género de España ${ }^{6}$ (1/2004 de 28 de diciembre):

Es ilícita la publicidad que atente contra la dignidad de la persona o vulnere los valores y derechos reconocidos en la Constitución, especialmente a los que se refieren sus artículos 18 y 20, apartado 4. Se entenderán incluidos en la previsión anterior los anuncios que presenten a las mujeres de forma vejatoria, bien utilizando particular y directamente su cuerpo o partes del mismo como mero objeto desvinculado del producto que se pretende promocionar, bien su imagen asociada a comportamientos estereotipados que vulneren los fundamentos de nuestro ordenamiento coadyuvando a generar la violencia a que se refiere la ley Orgánica de medidas de protección integral contra la violencia de género (España, Jefatura de Estado, 2004).

Uno de los objetivos fundamentales del ACD es observar las relaciones entre los textos y el poder social que los rodea; de ahí que el análisis llevado a cabo nos haya permitido establecer relaciones entre los textos y las normas y valores de la comunidad en que estos se enmarcan. Es decir, el contexto que enmarca a los textos estudiados es una sociedad patriarcal, androcéntrica y fetichista, en la que los cuerpos de las mujeres se conciben como objetos de deseo, hecho que contribuye a perpetuar una sociedad sexista basada en marcadas diferencias entre mujeres y hombres. A pesar de

6 Hay otros países con leyes similares. Se cita la ley española, por ser una de las primeras en las que el tipo de publicidad al que se hace referencia en este artículo se considera violencia simbólica. Conviene matizar que la marca es italiana, pero tiene presencia en todo el mundo. estar en el siglo XXI, es necesario seguir avanzando en la igualdad de género y en la necesidad de superar la violencia simbólica relacionada con el ideal de belleza femenina y los cuerpos de las mujeres. Por esta razón, es importante transmitir valores no sexistas en los textos que la publicidad genera, pues estos persisten en el pensamiento hegemónico de las sociedades actuales.

Las mujeres han de pasar de ser objetos pasivos en las representaciones a ser sujetos activos. Sus cuerpos han de ser representados en su totalidad y ha de evitarse cualquier muestra que lleve consigo la cosificación o el fomento del cuerpo como reclamo sexual o publicitario, al resaltar la dimensión erótica de la figura femenina. Se ha observado en las imágenes analizadas la objetivación del cuerpo femenino y el modo en que se hace uso de este recurso para establecer relaciones de poder y dominación.

\section{Conclusiones}

El hecho de que el cuerpo femenino esté tan cosificado e instrumentalizado en la publicidad analizada contribuye a reproducir patrones de violencia simbólica, que se normalizan y que perpetúan la publicidad sexista. Las formas de violencia simbólica estudiadas constituyen un ejemplo de subordinación femenina. Estas suponen un orden simbólico cultural en el que está presente la dominación masculina.

Dolce \& Gabbana utiliza los cuerpos de las mujeres como reclamo publicitario y como una figura sexualmente objetivada. La representación de las mujeres en posturas eróticas y provocativas no solo no favorece la igualdad de género, sino que además contribuye a la violencia simbólica y a los estereotipos de género. También se observa una tendencia a fragmentar los cuerpos de las mujeres y a destacar las zonas erógenas del pecho y los genitales, hecho que contribuye tanto a la violencia simbólica como a la cosificación del cuerpo.

El empleo de los cuerpos de las mujeres como reclamo publicitario por parte de Dolce \& Gabbana 
va unido a la dominación de los cuerpos, a destacar el poder que se ejerce sobre el cuerpo femenino en la publicidad, al usar el cuerpo con fines comerciales. Esto nos lleva a afirmar que nos encontramos ante formas de violencia simbólica normalizada. Consideramos que la misma hiperrepresentación en sí es otra modalidad de violencia simbólica. Por tanto, se debe repensar la categoría de "cuerpo" y los modelos culturales que se asocian con los cuerpos de las mujeres.

\section{Referencias}

Ananda, R., Fitriani, S. S., Samad, I. A., y Patak, A. A. (2019). Cigarette advertisements: A systemic functional grammar and multimodal analysis. Indonesian Journal of Applied Linguistics, 8(3), 616-626. https://doi.org/10.17509/ijal.v8i3.15261

Aparicio Martín, E. (2015). Los medios de comunicación en la violencia contra las mujeres: el paradigma de la delgadez. Historia y Comunicación Social, 20(1), 107 119. https://doi.org/10.5209/rev_HICS.2015.v20. $\mathrm{n} 1.49550$

Baykal, N. (2016). Multimodal construction of female looks: An analysis of mascara advertisements. Dilbilim Araşttrmaları Dergisi, 27(2), 39-59.

Blanco, J. (2009). Rostros visibles de la violencia invisible. Violencia simbólica que sostiene el patriarcado. Revista Venezolana de Estudios de la Mujer, 14(32), 63-70.

Bourdieu, P. (1998). La domination masculine. Éditions du Seuil.

Bourdieu, P. (2000). La dominación masculina. Anagrama.

Bowcher, W. L. (Ed.) (2012). Multimodal texts from around the world. Cultural and linguistic insights. Palgrave. https://doi.org/10.1057/9780230355347

Bringas López, A. (2010). Oscuros objetos de deseo: construcciones culturales del cuerpo femenino negro en el discurso publicitario. En B. Martín Lucas (Ed.), Violencias (in) visibles. Intervenciones feministas frente a la violencia patriarcal (pp. 115-138). Icaria.

Buonanno, M. (2014). Gender and media studies: Progress and challenge in a vibrant research field. Análisi. Quaderns de Comunicació i Cultura, (50), 5-25. http://dx.doi.org/10.7238/a.v0i50.2315

Cameron, D. (2006). On language and sexual politics. Routledge.
Coates, J. (2004). Women, men and language. A sociolinguistic account of gender differences in language (3. ${ }^{\mathrm{a}} \mathrm{ed}$.). Pearson.

Coates, J. (2012). Gender and discourse analysis. En J. P. Gee y M. Handford (Eds.), The Routledge handbook of discourse analysis (pp. 90-103). Routledge.

Cruz García, L. (2017). Evidentials in advertising: A sample study. Revista de Lingüistica y Lenguas Aplicadas, 12, 1-12. https://doi.org/10.4995/rlyla.2017.7074

Danilina, E. A., Kizyan, E. E., y Maksimova, D. S. (2019). Euphemisms in advertising discourse: Putting on a positive face and maintaining speech etiquette. Training, Language and Culture, 3(1), 8-22. https:// doi.org/10.29366/2019tlc.3.1.1

Del Rosso, T. (2017). There's a cream for that: A textual analysis of beauty and body-related advertisements aimed at middle-aged women, Journal of Women \& Aging, 29(2), 185-197. https://doi.org/10.1080/08 952841.2015 .1125698

Eckert, P., y McConnell-Ginet, S. (2003). Language and gender. Cambridge University Press. https://doi. org/10.1017/CBO9780511791147

España, Jefatura de Estado (2004). Ley orgánica de medidas de protección integral contra la violencia de género, 1/2004 de 28 de diciembre. Páginas 42166-42197. вОЕ 313 de 29/12/2004. Referencia 2004/21760. Ministerio de la Presidencia, Relaciones con las Cortes y Memoria Democrática, Gobierno de España. https://www.boe.es/eli/es/lo/2004/12/28/1

Fernández Fernández, P., Baños González, M., y García García, F. (2014). Análisis iconográfico de la publicidad audiovisual de perfumes. El caso J'Adore. Icono, 14(12), 398-430. https://doi.org/10.7195/ ri14.v12i1.549

Galarza, E., Cobo, R., y Esquembre, M. (2016). Medios y violencia simbólica contra las mujeres. Revista Latina de Comunicación Social, (71), 818-832. https:// doi.org/10.4185/RLCS-2016-1122

Giaccardi, S., Ward, M., Seabrook, R. C., Manago, A., y Lippman, J. (2016). Media and modern manhood: Testing associations between media consumption and young men's acceptance of traditional gender ideologies. Sex Roles, 75, 151-163. https://doi. org/10.1007/s11199-016-0588-z

Gil Calvo, E. (2000). Medias miradas. Un análisis cultural de la imagen femenina. Anagrama.

Goddard, A., y Patterson, L. M. (2000). Language and gender. Routledge. 
Halliday, M. A. K. (1978). Language as social semiotic. The sociological interpretation of language and meaning. Edward Arnold.

Halliday, M. A. K., y Matthiessen, C. M. I. M. (2004). An introduction to functional grammar (3. ${ }^{\mathrm{a}} \mathrm{ed}$.). Arnold.

Hashim, A., y Hassan, N. (2011). Genre analysis. En A. Hashim, D. Maya Khemlani y J. McLellan (Eds.), Text, discourse and society. Functional and pragmatic approaches to language in use (pp. 143-163). Peter Lang.

Jeffries, L. (2007). Textual construction of the female body. A critical discourse approach. Macmillan. https://doi. org/10.1057/9780230593626

Jerslev, A. (2017). The elderly female face in beauty and fashion ads: Joan Didion for Céline. European Journal of Cultural Studies, 21(3), 349-362. https://doi. org/10.1177/1367549417708436

Kress, G., y Van Leeuwen, T. (2006). Reading images: The grammar of visual design (2. ${ }^{\mathrm{a}} \mathrm{ed}$.) Routledge.

Magalhães, C. M., y Caetano, P. H. (2012). A comparative study of Brazilian and British images of the black body. En W. Bowcher (Ed.), Multimodal texts from around the world: Cultural and linguistic insights (pp. 299-324). Palgrave. https://doi. org/10.1057/9780230355347_13

Martín Lucas, B. (Ed.) (2010). Violencias (in)visibles. Intervenciones feministas frente a la violencia patriarcal. Icaria.

Martínez Lirola, M. (2009). Exploring new forms of gender violence: Women as objects in plastic surgery's leaflets. Revista Alicantina de Estudios Ingleses (RAEI), (22), 181-194. https://doi.org/10.14198/ raei.2009.22.12

Martínez Lirola, M. (2010). Explorando nuevas formas de violencia de género: la mujer como objeto en los folletos de clínicas de estética. Global Media Journal Mexico, 7(13), 80-94.

Martínez Lirola, M. (2012a). Deconstruyendo el discurso hegemónico del cuerpo perfecto: explorando la feminidad a través del análisis visual de folletos de clínica de estética. Feminismos, (19), 113-126.

Martínez Lirola, M. (2012b). Exploring the image of women to persuade in multimodal leaflets. Theory and Practice in English Studies, 5(1), 27-55.

Martínez Lirola, M., y Chovanec, J. (2012). The dream of a perfect body come true: Multimodality in cosmetic surgery advertising. Discourse and Society, 23(5), 487507. https://doi.org/10.1177/0957926512452970

Mora, N. (2014). Reproduction of patriarchal hegemony in media texts. Electronic Journal of Social Sciences, 13(48), 131-147.
Muñiz, E. (2010). Las prácticas corporales de la instrumentalidad a la complejidad. En E. Muñiz (Coord.), Disciplinas y prácticas corporales. Una mirada a las sociedades contemporáneas (pp. 1750). Anthropos.

Perdomo Colina, H. E. (2019). Aproximación discursiva a la construcción de la feminidad en el Concurso Nacional de Belleza de Colombia: 1977-2018. Cuestiones de género: de la igualdad y la diferencia, (14), 681-704. https://doi.org/10.18002/ cg.v0i14.5845

Pham, T. T. (2014). Moroccan immigrant women in Spain. Honor and marriage. Lexington Books.

Richardson, J. E. (2007). Analysing newspapers. An approach from critical discourse analysis. Macmillan.

Sarkar, S. (2014). Media and women image: A feminist discourse. Journal of Media and Communication Studies, 6(3), 48-58. https://doi. org/10.5897/JMCS2014.0384

Sato, T. (2011). Representation of desire and femininity. The advertisement in late-modern consumer culture of Japan. En D. Majstorović e I. Lassen (Eds.), Living with patriarchy. Discursive constructions of gendered subjects across cultures (pp. 145-167). John Benjamins. https://doi.org/10.1075/dapsac.45.09sat

Shaikh, M., Ali Bughio, A., y Ali Kadri, S. (2016). The representation of men and women in advertisements: A critical discourse analysis. Annual Research Journal of Gender Studies, 7, 108-141.

Sharda, A. (2014). Media and gender stereotyping: The need for media literacy. International Research Journal of Social Sciences, 3(8), 43-49.

Soley Beltran, P. (2015). ¡Divinas! Modelos, poder y mentiras. Anagrama.

Talbot, M. (2010). Language and gender (2. ${ }^{a}$ ed.). Polity Press.

Van Dijk, T. A. (2009). Critical discourse studies: A sociocognitive approach. En R. Wodak y M. Meyer (Eds.), Methods of critical discourse analysis (pp. 62-86). (2. ${ }^{a}$ ed.). Sage.

Van Leeuwen, T. (2008). Discourse and practice. New tools for critical discourse analysis. Oxford University Press. https://doi.org/10.1093/acprof:o so/9780195323306.001.0001

Velandia-Morales, A., y Rincón, J. C. (2014). Estereotipos y roles de género utilizados en la publicidad transmitida a través de la televisión. Universitas Psychologica, 13(2), 517-527. https://doi.org/10.11144/Javeriana.UPSY13-2.ergu 
Verdú Delgado, A.D. (2018). El sufrimiento de la mujer objeto. Consecuencias de la cosificación sexual de las mujeres en los medios de comunicación. Feminismo/s, (31), 167-186. https://doi.org/10.14198/fem.2018.31.08

Walter, N. (2010). Muñecas vivientes. El regreso del sexismo. Turner Publicaciones.

Wodak, R. (Ed.) (1997). Gender and discourse. Sage Publications.
Wodak, R. (2011). Critical discourse analysis. En K. Hyland y B. Paltridge (Eds.), The Continuum companion to discourse (pp. 38-53). Continuum.

Xu, H., y Tan, Y. (2020). Can beauty advertisements empower women? A critical discourse analysis of the SK-II's "Change Destiny" campaign. Theory and Practice in Language Studies, 10(2), 176-188. https://doi.org/10.17507/tpls.1002.05

Cómo citar este artículo: Martínez-Lirola, M. (2021). Violencia simbólica contra la mujer en los anuncios publicitarios: un análisis visual crítico. Íkala, Revista de Lenguaje y Cultura, 26(2), 349-364. https:// doi.org/10.17533/udea.ikala.v26n2a06 